\section{Nouvelles de l'industrie}

Neues aus der Industrie

\section{Recueil ouvert de données d'applica- tion de Remederm Spray Huile corporelle Louis Widmer}

\section{Traitement de soins en cas d'eczéma atopique}

Médecins responsables:

Dr A. Stepanova, Dr R. Disch

Directeur: Prof. Dr Dr phil.

S. Borelli

Alexanderhausklinik Davos, 2001

\section{Introduction}

L'eczéma atopique s'accompagne généralement de pertes hydriques élevées, d'un déficit de divers lipides cutanés et de la sébostase qui s'ensuit.

Il est d'autant plus important d'avoir régulièrement recours à des produits externes surgras. Ce faisant, les soins de la peau devraient intervenir non seulement à la phase aiguë, mais être poursuivis dans les intervalles pauci- resp. asymptomatiques.

Dans le cadre d'un recueil ouvert de données d'application, on a cherché à évaluer la tolérance et l'effet total de Remederm Spray Huile corporelle.

\section{Méthode}

L'observation a porté sur un total de 50 patients atteints d'eczéma atopique de degrés de sévérité divers. Il s'agissait de 32 femmes (64\%) et de 18 hommes (36\%) âgés de 18 à 66 ans. La durée d'observation a été de 4 semaines.

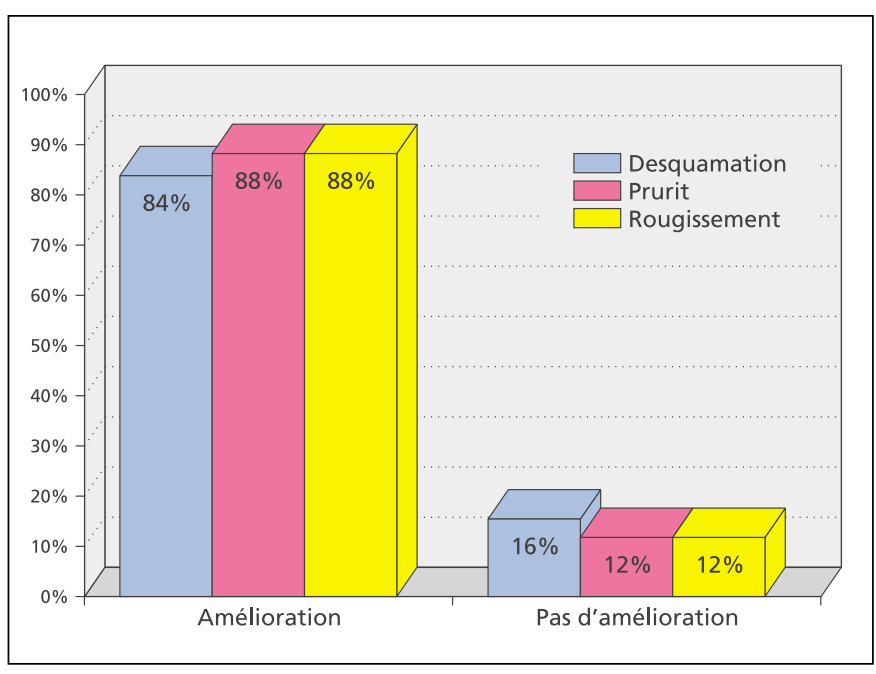

Fig. 1. Efficacité de Remederm Spray Hule corporelle

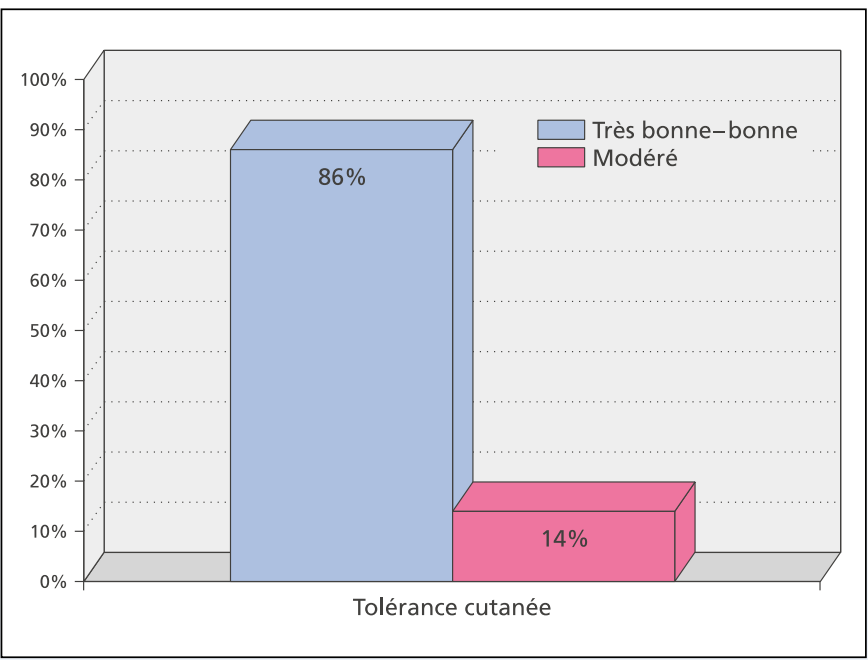

Fig. 2. Tolérance de Remederm Spray Huile corporelle.
Remederm Spray Huile corporelle a été appliquée 1 fois par jour.

Durant la période d'application, les patients et les médecins traitants ont été appelés à évaluer les symptômes «érythème», «prurit» et «desquamation».

\section{Résultats}

Les résultats de cette étude démontrent une très bonne efficacité de la préparation mise à l'essai. C'est ainsi que les symptômes cliniques de l'eczéma atopique ont été améliorés, pour l'érythème dans $88 \%$ des cas, pour la desquamation dans $84 \%$ des cas, pour le prurit dans $88 \%$ des cas (fig. 1).

Quant à la tolérance de Remederm Spray Huile corporelle, elle obtient la cote «très bonne» ou «bonne» dans $86 \%$ des cas (43 patients sur 50; fig. 2).
Chez les 7 patients restants, on a observé des réactions d'intolérance telles qu'érythème, prurit ou sensation de tension. C'est ce qui a fait arrêter l'application de la préparation chez 6 patients.

La bonne efficacité et la bonne tolérance ainsi documentées font chaudement recommander Remederm Spray Huile corporelle pour le traitement de l'eczéma atopique. Le produit contribue à améliorer la xérose cutanée, l'érythème, la desquamation et le prurit, apportant ainsi une contribution valable au succès thérapeutique.

Louis Widmer SA

Rietbachstrasse 5

CH-8952 Schlieren

Tél. 017301155

Fax 017302015

www.louis-widmer.com 
Offene

\section{Anwendungs-} beobachtung von Remederm Körperöl Spray

\section{Louis Widmer}

\section{Pflegende Behandlung bei Neurodermitis constitutionalis atopica}

Leitung: Dr. A. Stepanova,

Dr. R. Disch

Direktor: Univ.-Prof. Dr. Dr. phil. S. Borelli

Alexanderhausklinik Davos, 2001

\section{Einleitung}

Die Neurodermitis geht in der Regel mit einem erhöhten Wasserverlust, einem Verlust an verschiedenen Hautlipiden und einer daraus resultierenden Sebostase einher.

Deshalb ist es wichtig, dass man regelmässig rückfettende Externa verwendet. Dabei sollte die Hautpflege sowohl im akuten Stadium als auch in den symptomarmen bzw. symptomfreien Intervallen durchgeführt werden.

Im Rahmen einer offenen Anwendungsbeobachtung sollte der Remederm Körperöl Spray auf Verträglichkeit und Gesamtwirkung hin beurteilt werden.

\section{Methode}

In die Beobachtung wurden insgesamt 50 Patienten mit Neurodermitis constitutionalis atopica verschiedener Schweregrade aufgenommen. Dabei handelte es sich um 32 Frauen (64\%) und 18 Männer (36\%) im Alter von 18 bis 66 Jahren. Der Beobachtungszeitraum erstreckte sich über 4 Wochen.

Der Remederm Körperöl Spray wurde 1 mal täglich angewendet.

Dabei wurden die Symptome «Hautrötung», «Juckreiz» und «Schuppung» vom Patienten und vom Arzt beurteilt.

\section{Ergebnisse}

Die Untersuchungsergebnisse haben eine sehr gute Wirksamkeit des Prüf-

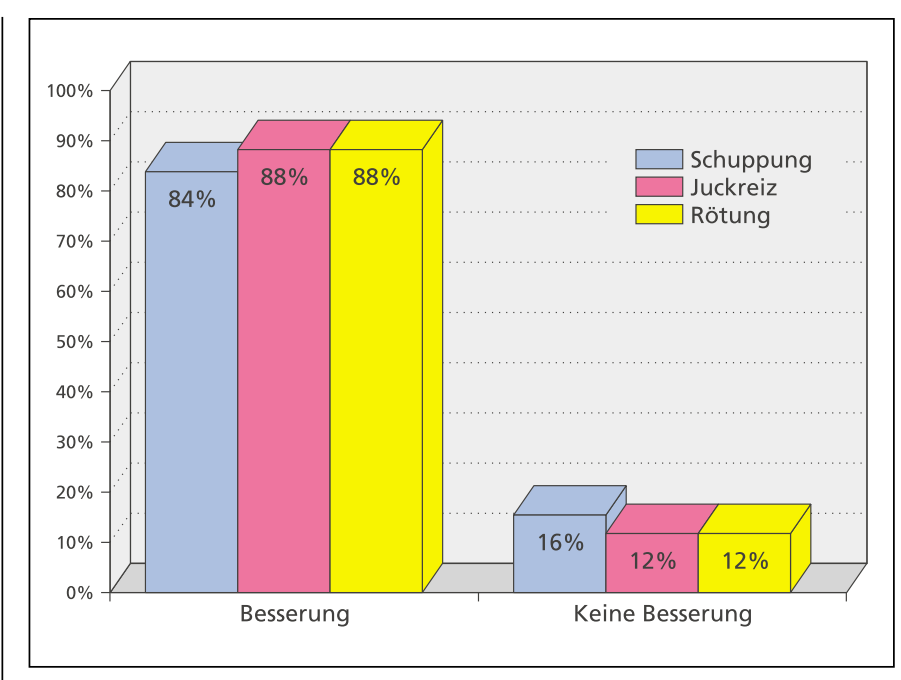

Abb. 1. Wirksamkeit von Remederm Körperöl Spray.

Abb. 2. Verträg-

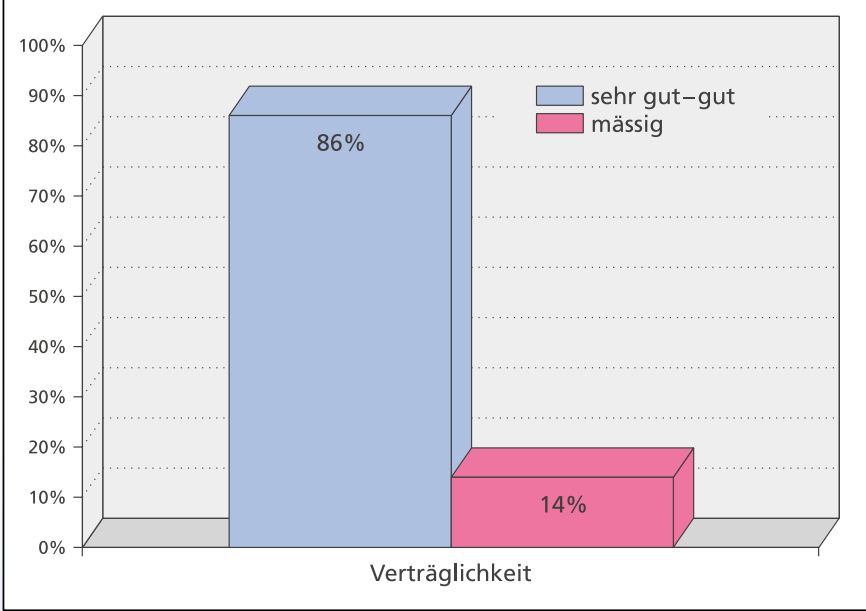
lichkeit von Remederm Körperöl Spray. präparates bewiesen. So konnte die klinische Symptomatik der Neurodermitis, wie Rötung in $88 \%$ der Fälle, Schuppung in $84 \%$ der Fälle und Juckreiz ebenfalls in $88 \%$ der Fälle, gebessert werden (Abb. 1).

Die Prüfung der Verträglichkeit des Remederm Körperöl Sprays ergab in $86 \%$ der Fälle (43 von 50 Patienten) «sehr gute» und «gute» Verträglichkeit (Abb. 2).

Bei den übrigen 7 Patienten wurden Unverträglichkeitsreaktionen, wie Rötung, Juckreiz und Spannungsgefühl, beobachtet. Deswegen haben 6 dieser Patienten die Anwendung des Prüfpräparates abgebrochen.

Durch die erwiesene gute Wirksamkeit und Verträglichkeit kann der Remederm Körperöl Spray sehr gut zur Behandlung der Neurodermitis empfohlen werden. Er trägt zur Verbesserung der
Hauttrockenheit, Hautrötung, Schuppung und des Juckreizes und somit zu einem Therapieerfolg bei.

Louis Widmer AG

Rietbachstrasse 5

CH-8952 Schlieren

Tel. 017301155

Fax 017302015

www.louis-widmer.com 